$\frac{p}{\frac{1}{0}}$

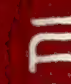

$\frac{0}{\frac{2}{2}}$

니

F

$\frac{1}{5}$

z

$\bar{A}$

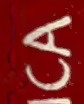

$\frac{1}{0}$

$\pi$

$\frac{1}{2}$

1

E

jo

J

$+$

3

3 


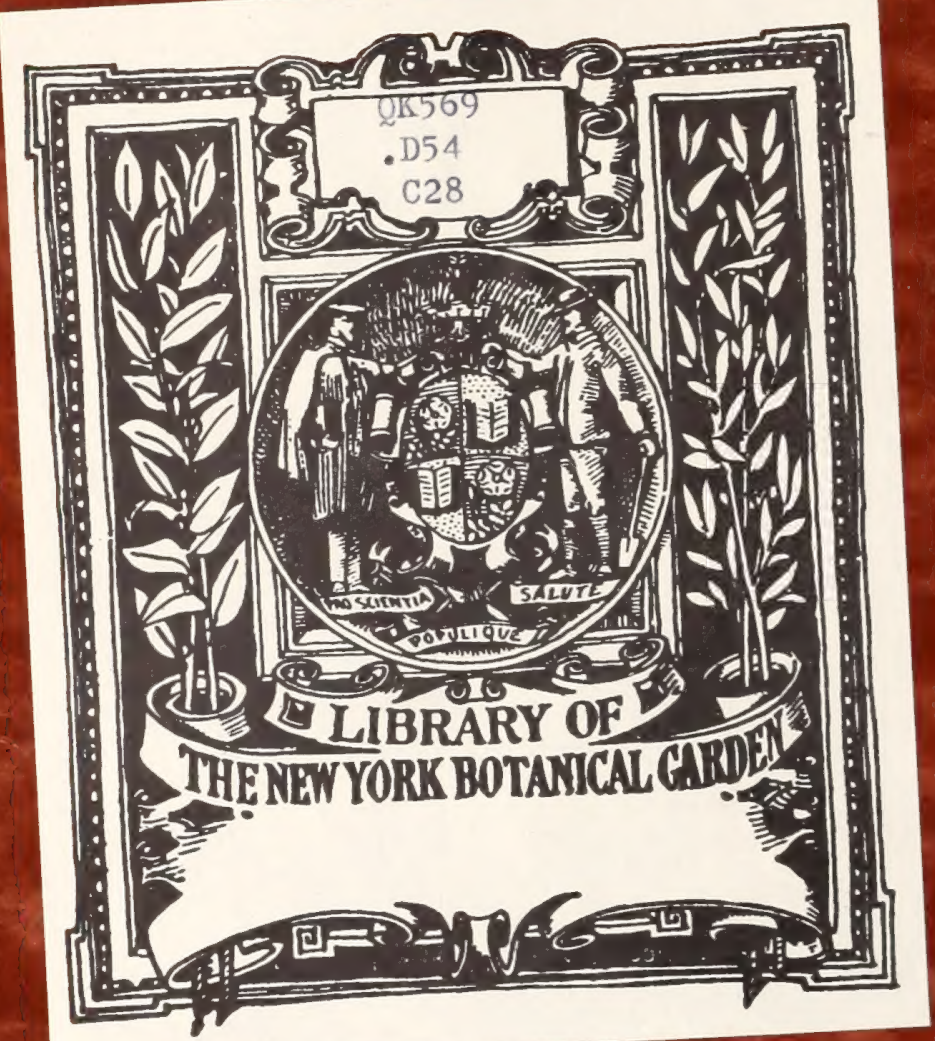




\title{
ANALISI MICROSCOPICA
}

\section{UN CALCARE}

\author{
DEL TERRITORIO DI SPOLETO
}

NOTA

DEL CONTE AB. FRANGESCO CASTRACANE DEGLI ANTELMINELLI

ESTRATTO DAGLI ATTI DELL'ACCADEMIA PONTIFICIA DENUOVI LINCEI ANNO XXXVIII - TOMO XXXVIII, SESSIONE IV DEL 15 MARZO 1885.

ROMA

TIPOGRAFIA DELLE SCIENZE MateMatiche B FISICHE

Via Lata, N." 3.

1886 


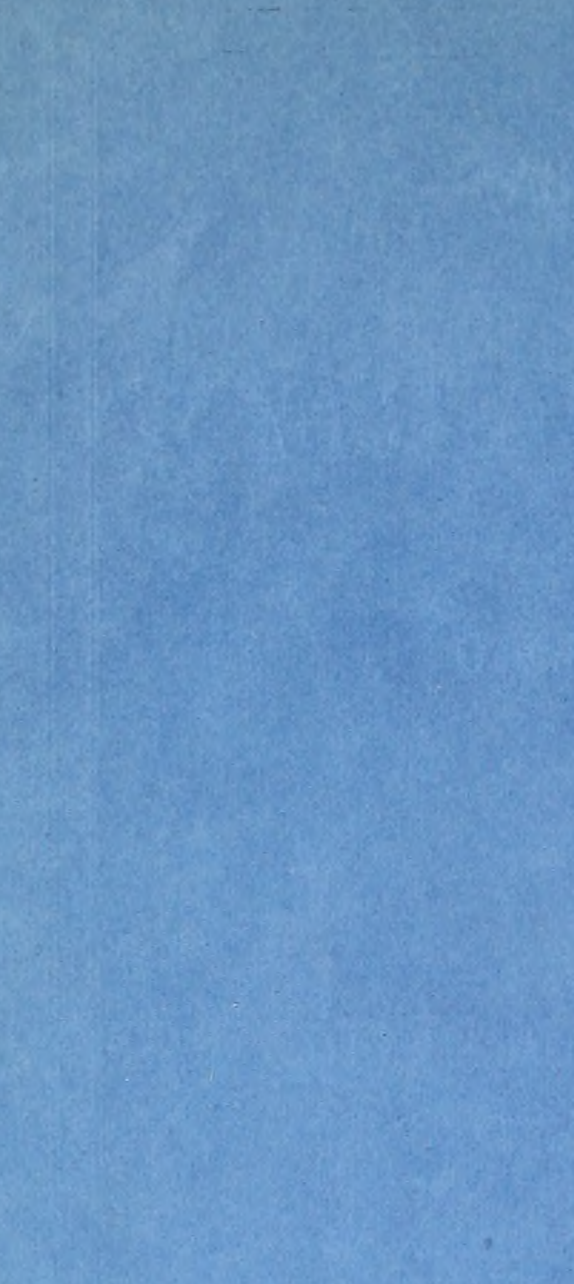




\section{ANALISI MICROSCOPICA}

\section{UN CALCARE}

\section{DEL TERRITORIO DI SPOLETO}

\section{NOTA}

DEL CONTE AB. FRANGESCO CASTRACANE DEGLI ANTELMINELLI

ESTRATTO DAGLI ATTI DELL'ACCADEMIA PONTIFICIA DE'NUOVI LINCEI ANNO XXXVIII - TOMO XXXVIII, SESSIONE IV DEL 15 MAR7O 1885.

\section{ROMA}

TIPOGRAFIA DELLE SCIENZE MATEMATICHE E FISICHE Via Lata, $\mathrm{N} 0^{\circ} 3$.

1886 
.$D 54 C 28$

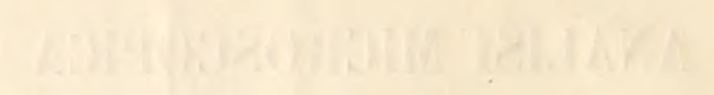

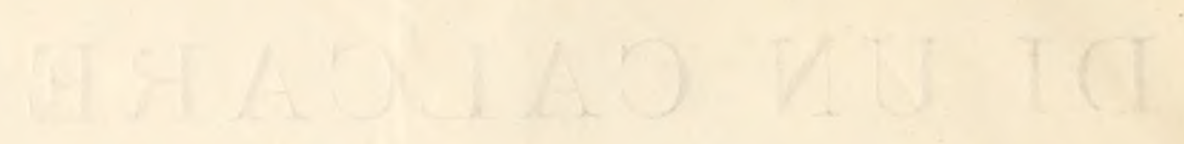

han (1) 


\section{ANALISI MICROSCOPICA}

DI UN GALGARE

DEL TERRITORIO DI SPOLETO

$\mathrm{N}_{\mathrm{el}}$ agionare della pratica utilità dello studio delle Diatomee, e delle diverse possibili applicazioni di quello ai molteplici rami della Biologia e Storia Naturale, più volte io ho avuto occasione di ricordare in particolar modo quanto valido aiuto da quello studio potrebbe trarre il Geologo a conoscere la storia di quelle formazioni ove le Diatomee vennero riconosciute. Fu pertanto ottimo consiglio quello dell'illustre Ehrenberg, quando intitolò la sua grande opera su le diatomee fossili Microgeologia, quantunque mi avrebbe sembrato meglio adatto il nome di Micropaleontografia, se pure non sonasse male la soverchia lunghezza di questo. Ed in fatti a rigore considerando la cosa non mi sembra appropriato l'aggettivo $\mu$ uxpos proposto al nome geologia perchè, quantunque si argomenti da minimi organismi, il giudizio viene formulato su la storia di uno strato di un banco di un giacimento di notevolissima estensione; invece il termine di nicropaleontologia conviene a quello studio che versa attorno minutissimi organismi, i quali poterono conservarsi o lasciar loro impronte dalle più remote epoche geologiche, e stanno ad attestare al Geologo le condizioni con le 
quali ebbe luogo quella formazione. Se pertanto fosse bene impresso nella mente dei Geologi Italiani l'importanza somma, che ha per la conoscenza della storia della formazione della nostra bella penisola il tenere conto dei minini organismi, dall'enorme agglomeramento dei quali vengono spesso costituiti gli strati, il Microscopio dovrebbe sempre aver luogo fra la supellettile del Geologo. Quantunque non si possa preiendere che il Geologo sia al caso di poiere determinare ogni Diatomea o Radiolaria o Policistina, ciò che assorbirebbe troppo tempo, ̀̀ però indispensabile a mio modo di vedere che senza potere esattamente determinare il genere e la specie di ciascuno di quei minimi organismi, sia però in grado di costatarne la presenza, ricorrendo quindi al Micrografo specialista perchè convenientemente li determini. Il Geologo potrá essere ben persuaso che ove Esso potè appena constatare qualche traccia di corpicciuoli organizzati quegli che ne fece speciale soggetto di studio per l'abitudine e la lunga esperienza acquistata in tali ricerche, potrà con relativa facilità, isolarne un grande numero, cosi che ne rimarra maravigliato per la copia, avendone prima intraveduto appena qualche traccia.

Fra i Geologi Italiani, che meglio si mostrarono persuasi della importanza che offrono le Diatomee ad indagare la storia della nostra Penisola nelle epoche già remote merita precipua lode il Professore Dante Pantanelli, il quale riconosciuta la presenza di microrganismi nei terreni che andava studiando, più volte $\mathrm{mi}$ ha gentilmente favorito campioni di roccia e materiali da Esso raccolti. Intendo pertanto aitestare all'illustre Geologo la mia più viva riconoscenza per la gentilezza usatami con communicarmi quei materiali, avendo con ciò contribuito ad allargare le mie cognizioni su le Diatomee e più specialmente su i depositi Italiani. A Lui si deve l'avere indicato l'esistenza di un deposito di Dialomee e di Radiolarie nell'Appennino settentrionale e precisamente in quel di Modena presso Monte Gibbio e Baiso, e nel territorio Reggiano nella vicinanza della località detta i Quattro Castelli, già signoria della famosa Contessa Matilde e quindi della nobilissima famiglia dei Canossa. Fra i campioni diversi di quei depositi potei constatare l'esistenza di alcuni tipi, che sin ora non solamente non furono noverati fra le forme Italiche, ma talune non vennero pure ricordate nella flora Europea, e a convincere della verita di questo sarà sufficiente argomento il ricordare l'avere io riconosciuto in una preparazione del deposito di Monte Gibbio una nuova specie di Rutilaria, genere il più raro 
sin ora fra guanti si conoscono, del quale un esemplare costituisce uno dei più rari gioielli che può vantare una collezione.

Devo pure alla gentilezza del Dott. Pantanelli l'avere acquistato la cognizione di un nuovo deposito di acqua dolce esistente nel territorio di Spoleto nell'Umbria. Esso mi inviava un campione di marna, che mi diceva appartenere al pliocene inferiore, la quale marna era sovraincombente a un banco di lignite dello spessore di ben dieciotto metri, il quale viene ora scavato per utilizzare quel combustibile a pro' di diverse industrie. Alla domanda del cortesissimo Donatore volentieri assunsi l'impegno di determinare i generi e le specie di Diatomee ivi contenute, accingendomi subito all'impresa. Alla prima occhiata che sul il campo del microscopio gettai all'insieme del materiale subito mi apparvero numerose Epithemie e Cyclotelle, ma tutte rimanevano perdute fra abbondantissimo detrito roccioso e non poco materiale amorfo. Allora procedetti a tentare di liberare le Diatomee della maggiore quantità di quelle sostanze ingombranti trattando il materiale con acido solforico alquanto diluito, con il quale lo feci bollire in un provino, e atteso che ogni sostanza carboniosa venisse ossidata (ciò che veniva indicato dall'annerire del liquido), con l'aggiunta di piccolissimi cristallini di clorato potassico su l'acido bollente si avevano delle decrepitazioni, e in pari tempo dileguavasi interamente il colore nero nel provino, avendo luogo in quello la più completa disossidazione. A questo punto gettato il liquido acido e il materiale contenuto nell'acqua, procedevo a numerosissime accurate decantazioni ad eliminarne l'acido ed $\mathrm{i}$ sali solubili e quindi con ripetute lavande in acqua distillata purissima e con successive graduali levigazioni pervenni a fare belle preparazioni di Diatomee quasi perfettamente libere dalla associazione di sostanze estranee.

Ottenuto per tal modo di avere buone preparazioni di quel deposito, senza grave difficoltà procedetti a determinarne i tipi generici e specifici che vi si contengono. Come già dissi le forme più frequenti ad incontrare in quel deposito sono le Epithemie e le Cyclotelle. Le prime attraggono lo sguardo per la grandezza dei loro frustoli, e alla prima occhiata si riconosce esistere fra un tipo e l'altro notevoli differenze, che li dimostrano appartenenti a specie distinte. Ma la bellezza e il grande numero delle Cyclotelle richiamò principalmente la mia attenzione. Queste mi si presentavano sotto l'aspetto di elegantissimi dischi di diverse grandezze; ciascuno mostravasi contornato da larga fascia radialmente striata, larga circa un terzo del raggio. Una tal fascia circoscrive l'area centrale, che vedesi or- 
nata da una stella di belle perle disposte quasi regolarmente a raggi con alcuni puntini qua e là disseminati. Un tale tipo specifico di Cyclotella ho posto a confronto con le diverse figure e relative descrizioni che abbiamo riguardanti un tale genere. La larga fascia radialmente striata con stria più forte ricorrente a regolari intervalli la ravvicina alla $C_{y}$. comta. E. Kz. ma ne differisce completamente nella striazione o granulazione dell'area media. Ed in fatti nella Cy. comia, il centro è finamente striato, a strie stipate più o meno radianti: invece nel ípo da noi preso in esame, l'area è elegantemente decorata da perle o grossi e rari granuli in file radianti, in distribuzione meno regolare, notandovisi contemporaneamente interpolati senza alcun ordine alcuni piccolissimi granuli puntiformi. Conosco ancora la $C y$. operculata, Kz. var. radiosa, Grunow, la quale ha l'area centrale striata, ma in questa non si riscontra il carattere dei grossi e rari granuli, uè l'interpolazione irregolare di granuli piccolissimi puntiformi. Se avrei esitato nel riconoscere nella Diatomea Umbra una nuova forma specifica, incontrandone soltanto alcun raro esemplare, però non mi può rimanere alcun dubbio od esitazione nel caso nostro, avendone avanti gli occhi infinito numero di esemplari dotati tutli di identici caratteri. Dovendo pertanto alla gentilezza del Pantanelli la cognizione di questo nuovo elegantissimo tipo nella relativa ristrettezza della flora diatomacea di acqua dolce tanto più accessibile alle nostre ricerche e conseguentemente tanto più cognita della flora marina, tale nuova forma specifica sarà ricordata con il nome di Cy. Pantanelliana Cstr. ed eccone la definizione.

Cy, Pantanelliana. Cstr. nova species. "E maximis : a latere rectangula, a fronte plana, tertia radii parte circum radiata ; area centrali rariusculis margaritis subregulariter radiata, nonnullis punctulis interpolatis. "

Le forme diverse di Diatomee sin ora da me determinate nel materiale Spoletino sono le seguenti:

Epithemia Hyndmannii. W. Sm.

Zebra, Kz.

" ocellata, $\mathrm{Kz}$.

„ proboscidea, $\mathrm{Kr}$.

Cocconeis Placentula. E.

Cyclotella Pantanelliana. Cstr. n. sp.

Cymbella cuspidata. Kz.

„ obtusiuscula, Kz.

y. gastroides, $\mathrm{Kz}$. 
" (Cocconema) Cistula, E.

Pinnularia acuta, W. Sm.

$\checkmark$ radiosa, W. Sm.

Navicula ovalis. W. Sm.

Fragilaria (Odontidium) Harrisonii, W. Sm.

Gomphonema Vibrio. E.

"? curvatum. Kz.

Con questo breve elenco di Diatomee non intendo per alcun modo indicare che quelle siano le sole forme racchiuse in quel deposito, non essendomi in verun modo proposto il redigere un lavoro monografico, mentre invece volli solianto dare un saggio dei tipi ivi inclusi, ricordando in particolar modo i tipi che sono dominanti in quel materiale, e che gli imprimono una speciale fisonomia. Tali sono sopra tutte le diatomee le numerosissime Cyclotelle, e le forme diverse di Epithemia. Però oltre quelle merita speciale menzione la Fragilaria (Odontidium) Harrisonii. W. Sm. e per l'eleganza dei suoi frustuli cruciformi, e per la rarita di tale tipo. Questo da principio venne dall'illustre W. Smith (Synopsis of the Britisch Diatomacee, II, pag, 18) con qualche riserva denominato Odontidium? Harrisonii, notando però che i caratteri forse potevano farlo riguardare come appartenente al genere Staurosira. E. In seguito il D.r Rabenhorst avendo riunito il succitato genere alla Fragilaria ritenne il tipo Smithiano riguardandolo come Fragilaria Harrisonii, quantunque la disposizione a file a zig zag avreble meglio dovuto ascriverlo alle Tabellarie (Rabenhorst=Flora algarum Europearum, vol. I. pag. 18.) Questa Diatomea e forse la nuova specie di Cyclotella, per la di cui presenza il deposito, che ho preso ad esame, dovra essere ricordato fra i più interessanti depositi di acqua dolce. Nè potrebbe aver luogo alcun dubbio nel riguardare quel deposito prettamente di acqua dolce, non essedovi tipo alcuno, il quale non venga di leggieri riconosciuto come proprio della flora terrestre, come apparirà a chiunque sia appena famigliarizzato con la conoscenza di tali organismi. Ma, quando lo sludio di questi minimi organismi verrà seguito con serieta di propositi, possiamo essere certi, che dall'esame delle forme dialomacee, che si riconosceranno far parte di alcun deposito si potranno dedurre più particolarizzate notizie su le circostanze, che esercitarono la loro influenza su quella formazione. Trovo registrato nelle mie memorie l'avere per più anni raccolto abondantemente l'Achnantes ventricosa. E. da una piccola sorgente in quel di Gubbio mentre in altre sorgive site nel medesimo terreno e a pochi metri di distanza mai potei fra 
le altre Diatomee incontrare un solo esemplare di quella rara specie. Chi potrà pertanto persuadersi che non vi sia a tale anomalia una ragione suficiente, sia della diversità dello strato, dal quale fluiscono quelle acque, sia da diversa costituzione chimica del terreno? L'illustre micrografo Alfonso de Prebisson mi diceva di avere osservato che il Gomphonema geminatum, Ag. suole incontrarsi inquelle località ove abonda il principio calcare. Ne manclierebbero altri esempi a dimostrare come circostanze locali di costituzione di terreno, condizioni di temperatura, relativa altezza sul lisello del mare e conseguente differenza di pressione barometrica siano meglio favorevoli alla vegetazione di una che di altra specie, cosichè dalla qualita delle Diatomee facenti parte di uno strato geologico si possa arguire le circostanze speciali, nelle quali tal formazione ebbe luogo. Intento a ricercare utili applicazioni dello studio al quale mi dedicai, volli vedere se dall'analisi microscopica istituita nel materiale Spoletino potessi divinare le circostcnze sull' influenza delle quali ebbe lnogo quel deposito; ponendo a confronto le mie deduzioni con le osservazioni stratigrafiche e peleontologiche che l'esimio Professore Pantanelli su la faccia del luogo aveva dovuto fare con più sicuro giudizio.

Indirizzatomi pertanto all' illustre Geologo per dargli conto delle forme diverse da me determinate in quel deposito, ed in pari tempo sottoponendogli le mie deduzioni perchè le cimentasse con i criteri geognostici da Esso raccolti, ecco quanto in proposito mi scrivea in data del 12 Marzo. "Ella si è apposto bene ritenendo cle le Diatomee Spoletine provennero " da un ampio lago; infatti appartengono alla vasta deposizione lacustre " della valle del Maroggia, che poi si continuava con quella di Foligno e " probabilmente con quella dell'alto Tevere : ivi lo spessore dei depositi " lacustri ascende a qualche centinajo di metri e $\mathrm{i}$ pochi molluschi ivi rac" colti confermano quello che Lei ha detto per le fliatomee. Il calcare o " marna calcarea a Diatomee è sovrapposta a un banco di lignite di is " metri di spessore. Non doveva poi essere come Ella dice molto elevato " sul livello del mare pliocenico 1: perchè la sua grande estensione male " si accorda con la orografia pliocenica, che non comportava in quel pe"riodo vaste estensioni di terre emerse. $2^{\circ}$. perchè il pliocene marino nella "valle del Tevere (S. Gemini, etc.) è ad una altezza superiore a qucsti de"positi. "

Non occorre certamente che io mi trattenga guari a dire qual piacere io provassi nel leggere guelle parole. L'assicurazione che il giudizio da me 
furnulato dalla ispezione e determinazione dei microorganismi esistenti in quel materiale fosse consentaneo al vero, la quale assicurazione autorevolissima veniva appoggiata ad argomenti del maggior peso dedotti dallo studio accurato della localita per parte dell'illustre Geologo e Paleontologo nel dovere dar conto dello scheletro di due Mastodonti e di un Tapiro rinvenuti fra quelle ligniti, mi convinceva essermi io bene apposto nel giudicare della importanza che può avere lo studio delle Diatomee per il progresso della Geologia. Un tale esempio di perfetta consonanza fra le osservazioni del Micrografo e le indicazioni stratigrafiche e paleontologiche, se non mi inganno, varà a persuadere il Gcologo della opportunita di rintracciare nel campo delle sue ricerche e péregrinazioni la presenza di minimi organismi, invocando alla circostanza l'aiuto dello specialista a meglio determinarli. Cosi pure il Micrografo dall'esempio addotto potrà essere eccitato a imprimere al proprio studio una direzione tale, che possa servire al progresso di altri rami di Scienza, se non anche a pratiche applicazioni a benefizio del civile consorzio.

E proprio delle verita tutte che più o meno dapresso l'una dall'altra dipendano come che sono emanazioni del sommo Vero. Cosi qualunque ramo di Storia naturale, che si prenda a coltivare, quando lo studio ne venga fatto con serietà di propositi, non tarderanno guari a far conoscere i legami che stringono l'uno all'altro cusichì siasi da attendere utili applicazioni da qualunque specialità, cui ci dedicammo. Queste applicazioni scaturiscono spontance a misura che veniamo meglio ad approfondire lo studio intrapreso, ricercandone le attinenze. Però l'ordine logico evidentemente richiede che la cognizione di una cosa preceda la sua utilizzazione, e non viceversa. Ma le Diatomee, le quali ci sono note soltanto da pochi lustri, cioè dal momento che specialmente per l'opera del Modenese Professorc Gio. Batt. ${ }^{a}$ Amici si potè ottenere ottimi microscopi acromatici, quelle fra le tante maraviglie del microcosmo ci vennero rivelate dal prezioso istrumento, ma non furono studiate se non che da pochi, e lo sludio che se ne fece per lo più non venne diretto se non che alla determinazione e descrizione dei diversi tipi. Non è pertanto da maravigliare se tutt'ora la Diatomologia venga riguardata quale studio di puro lusso. Che però tale non sia questo studio ritengo che fra li altri argomenti che potrebbero opportunamente addursi l'esempio citato valga a dimostrarlo in ordine alla Geologia. Possa una tale verità imprimersi nella mente di quanti con lodevolissimo impegno si dedicarono alla coltura di una Scienza, la quale ad onta che sia la piu giovane fra tutte ora grandeggia fra le Scienze sorelle.

F. Castracane. 



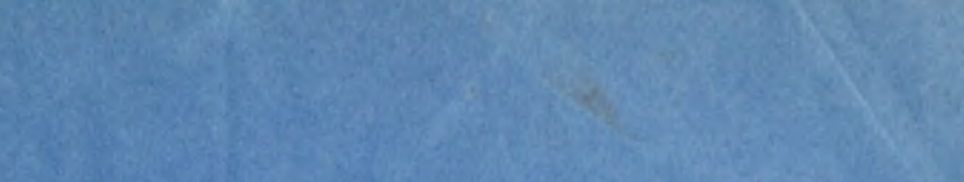

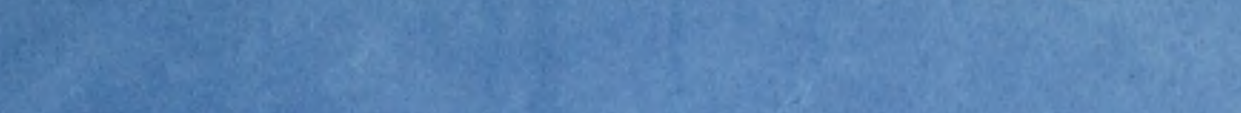

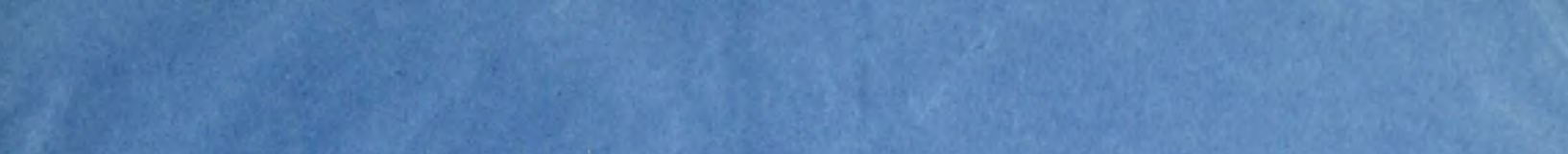

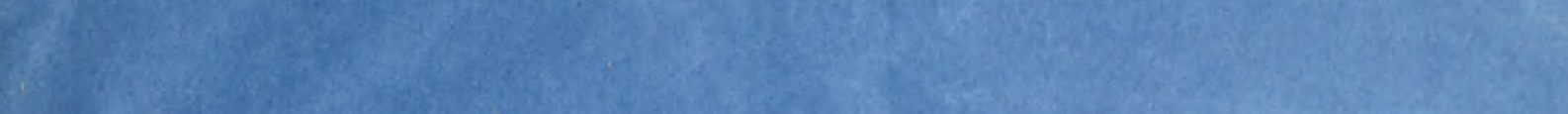
Als

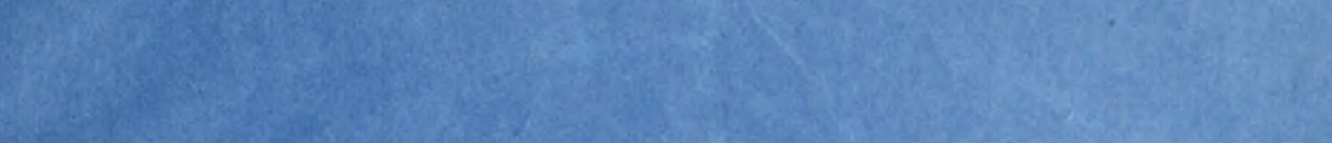

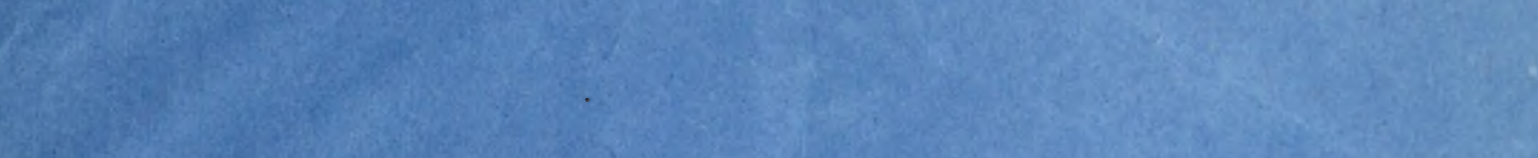
(3) (a) (x)

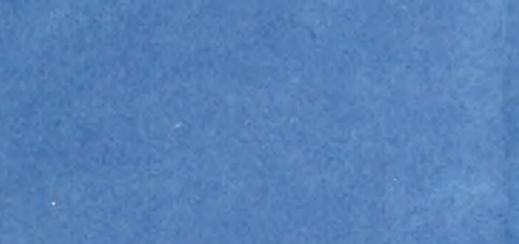

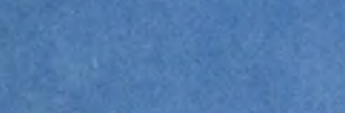

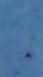

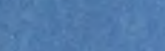

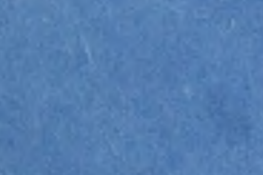

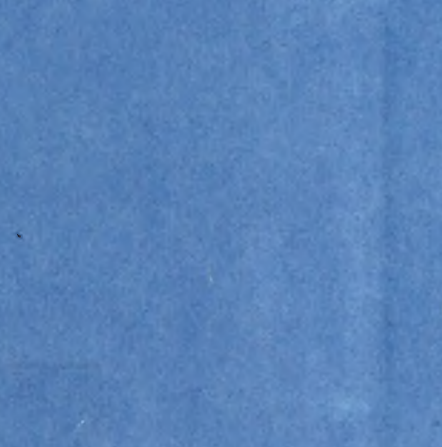

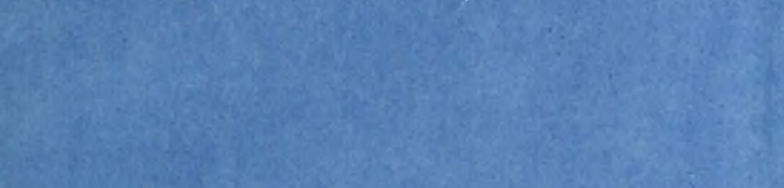

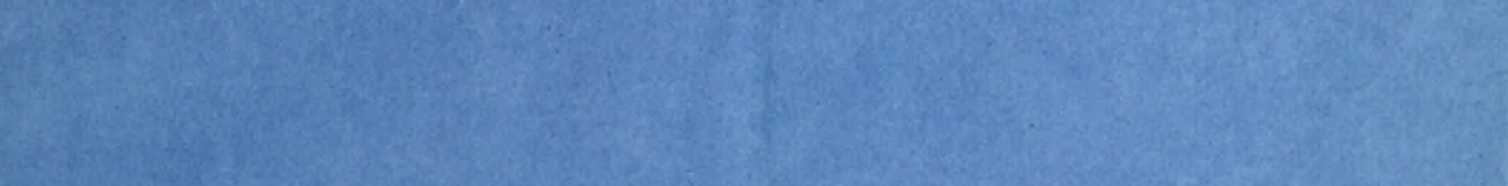

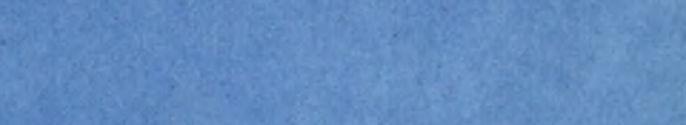

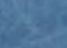
$x=$

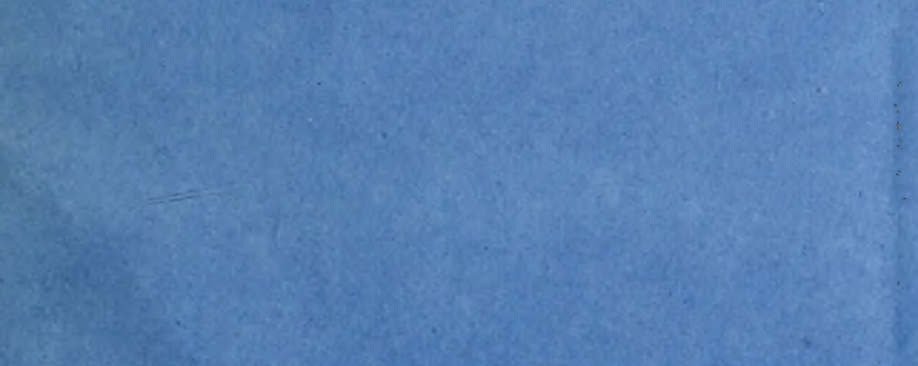

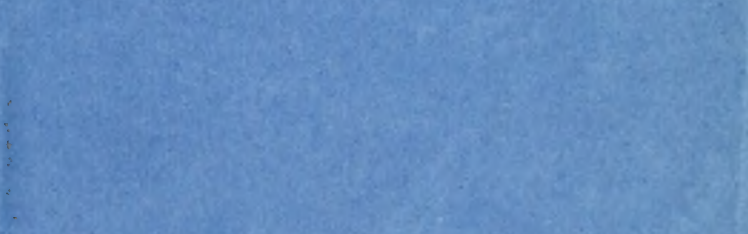

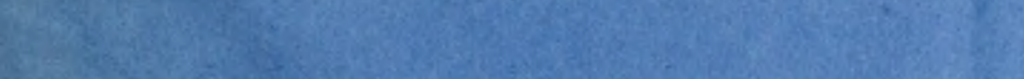

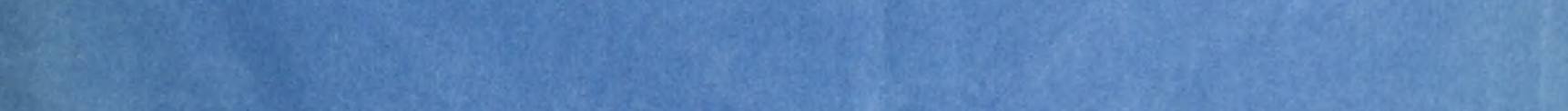
3.

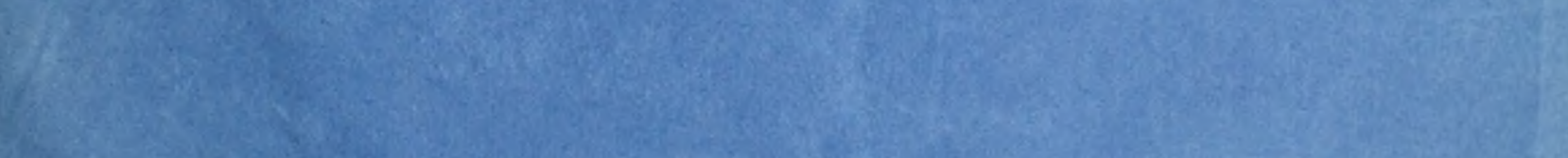

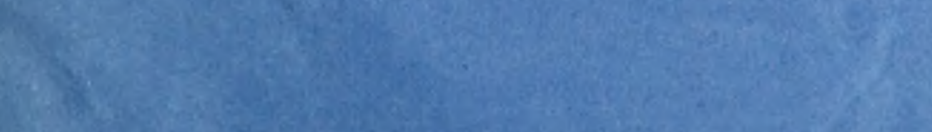

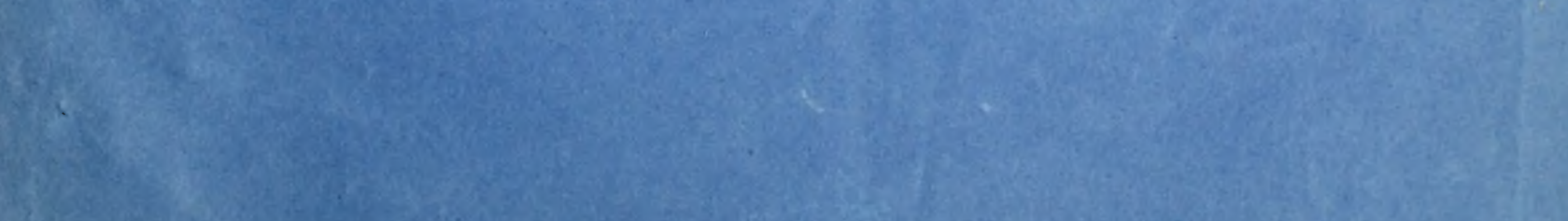




